\title{
Whole slide imaging in pathology: advantages, limitations, and emerging perspectives
}

This article was published in the following Dove Press journal:

Pathology and Laboratory Medicine International

II June 2015

Number of times this article has been viewed

\author{
Navid Farahani' \\ Anil V Parwani ${ }^{2}$ \\ Liron Pantanowitz ${ }^{2}$ \\ 'Department of Pathology and \\ Laboratory Medicine, Cedars-Sinai \\ Medical Center, Los Angeles, CA, USA; \\ ${ }^{2}$ Department of Pathology, University \\ of Pittsburgh School of Medicine, \\ Pittsburgh, PA, USA
}

\begin{abstract}
Significant technologic gains have led to the adoption of innovative digital imaging solutions in pathology. Whole slide imaging (WSI), which refers to scanning of conventional glass slides in order to produce digital slides, is the most recent imaging modality being employed by pathology departments worldwide. WSI continues to gain traction among pathologists for diagnostic, educational, and research purposes. This article provides a technologic review of WSI platforms and covers clinical and nonclinical pathology applications of these imaging systems. Barriers to adoption of WSI include limiting technology, image quality, problems with scanning all materials (eg, cytology slides), cost, digital slide storage, inability to handle high-throughput routine work, regulatory barriers, ergonomics, and pathologists' reluctance. Emerging issues related to clinical validation, standardization, and forthcoming advances in the field are also addressed.
\end{abstract}

Keywords: digital, imaging, microscopy, pathology, validation, whole slide image, telepathology

\section{Introduction}

The era of digital pathology continues to evolve at a rapid pace, primarily driven by developments in technology. Persistent gains in computer processing power, data transfer speeds, advances in software, and cloud storage solutions have enabled the use of digital images for a wide variety of purposes in pathology. As a result, pathology departments have begun utilizing digital imaging for simple tasks (eg, image archiving) and more complex undertakings (eg, telepathology, image analysis). ${ }^{1-9}$ Digital imaging modalities have shifted from using cameras to acquire static images toward whole slide imaging (WSI), which is a relatively novel technology. ${ }^{10}$

WSI, also commonly referred to as "virtual microscopy", 5 aims to emulate conventional light microscopy in a computer-generated manner. Practically speaking, WSI consists of two processes. The first process utilizes specialized hardware (scanner) to digitize glass slides, which generates a large representative digital image (so-called "digital slide"). The second process employs specialized software (ie, virtual slide viewer $)^{5,6}$ to view and/or analyze these enormous digital files. Figure 1 shows an example of a contemporary WSI scanner and virtual slide viewer. During the last decade, a wide range of commercially available WSI instruments have been developed. A list of common WSI systems and their respective vendors is provided in Table 1. These devices are meant to meet the needs of a diverse user base. A list of differences between selected WSI systems is provided in Table 2 .
Correspondence: Liron Pantanowitz Department of Pathology, UPMC Cancer Pavilion Suite 20I, 5I50 Centre Ave, Pittsburgh, PA I5232, USA

Tel + I 4126233746

Fax + I 4I26234779

Email pantanowitzl@upmc.edu 


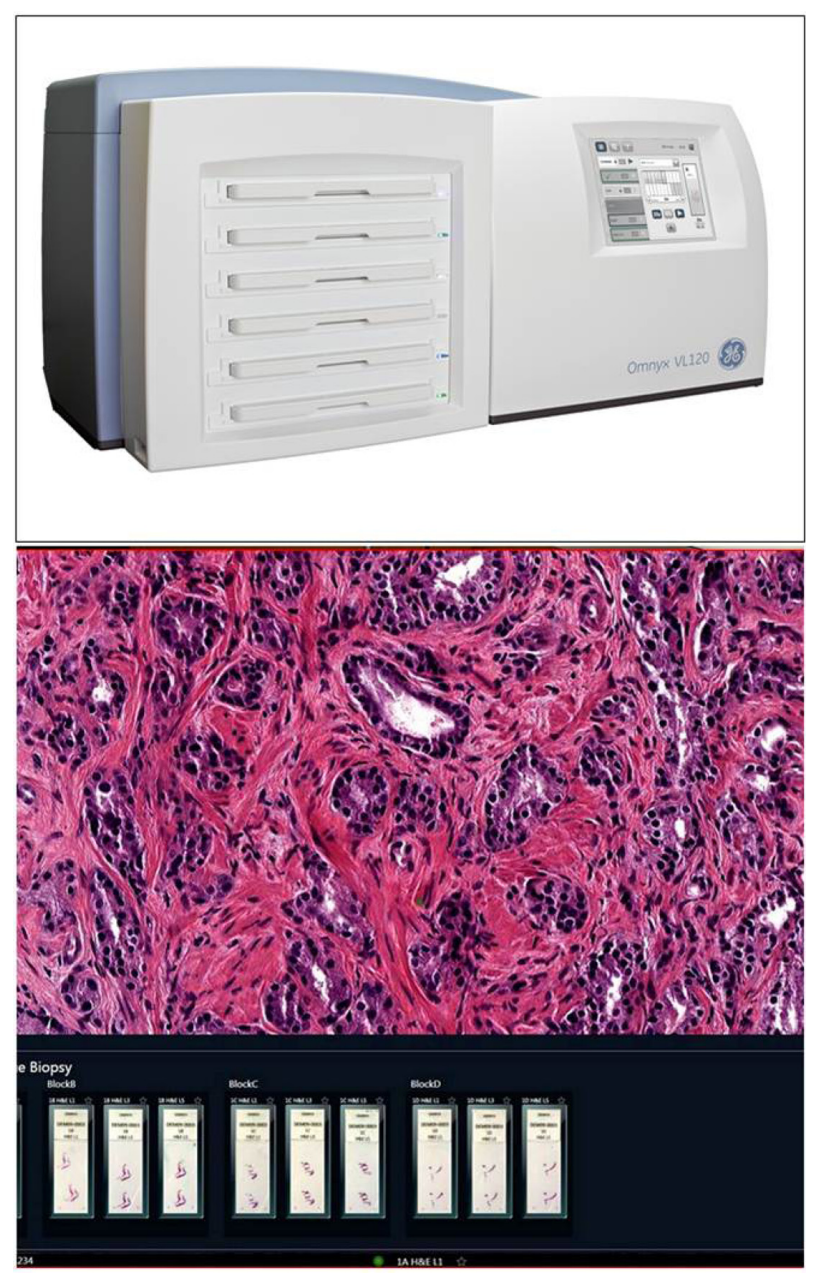

Figure I (Top) Omnyx whole slide imaging scanner. (Bottom) Omnyx viewer and integrated digital pathology solution that facilitates pathologist workflow. (Images courtesy of Omnyx).

The aim of this article is to review the technology and demonstrate the use of WSI for various clinical and nonclinical uses in pathology. Emerging issues related to clinical validation of these digital imaging systems and recent advances in the field are also addressed.

\section{History}

The first WSI scanners, introduced in the late 1990s, were quite primitive compared with their contemporary counterparts. ${ }^{7}$ Prior to the introduction of WSI, digital imaging in anatomic pathology relied largely on microscopemounted cameras to produce "static" digital images. ${ }^{2}$ These static images were of limited clinical utility because they captured only specific regions of a glass slide. Robotic microscopy was accordingly utilized because it allowed the telepathologist to remotely review an entire glass slide. ${ }^{11}$ Such stand-alone robotic systems are no longer vendor supported and are therefore infrequently being used today. Robotic microscopic capability, however, has begun to be
Table I Present-day commercially available whole slide imaging (WSI) scanners

\begin{tabular}{ll}
\hline Vendor & WSI scanner model \\
\hline 3DHistech & Pannoramic SCAN II, 250 Flash \\
DigiPath & PathScope \\
Hamamatsu & NanoZoomer RS, HT, and XR \\
Huron & TISSUEscope 4000, 4000XT, HS \\
Leica* & ScanScope AT, AT2, CS, FL, SCN400 \\
Mikroscan & D2 \\
Olympus & VSI20-SL \\
Omnyx & VL4, VLI20 \\
PerkinElmer & Lamina \\
Philips & Ultra-Fast Scanner \\
Sakura Finetek & VisionTek \\
Unic & Precice 500, Precice 600x \\
Ventana** & iScan Coreo, iScan HT \\
Zeiss & Axio Scan.ZI \\
\hline Notes: *Leica was formerly known and operated as Aperio; **Ventana was formerly \\
known and operated as Bioimagene.
\end{tabular}

incorporated into several WSI scanners. WSI technology was inspired by pioneering efforts to achieve high-resolution scanning of an entire glass slide. In 1997, Ferreira et a $1^{12}$ created a virtual microscope with the potential to capture large areas of a slide utilizing robotic microscopy. This system utilized a robot-microscope-computer combination to create a mosaic pattern of image tiles that produced a composite "slide image". While this early system was groundbreaking, it was limited by the length of time it took to scan a slide and the fact that it captured only a single extended field (commonly referred to as a "static image"). ${ }^{2}$ The next major development in WSI was followed by the advent of an automated, high-speed system created by Interscope Technologies. Their aim was to produce a system capable of capturing entire slides at high resolution but in a timeefficient fashion and with reasonable operating costs. ${ }^{13}$ Their success ushered in a new era, as soon thereafter a torrent of automated, affordable WSI scanners became commercially available. ${ }^{14,15}$ Most modern WSI instruments today are capable of producing high-resolution digital slides in the span of minutes (or less). ${ }^{7}$

\section{Technology}

\section{Hardware}

WSI devices have a wide range of appearances and functionality, geared to meet the needs of a large and diverse consumer market base. Some scanners have a small desktop footprint, designed to scan only a limited number of slides, while other larger instruments can accommodate hundreds of glass slides. Slides, including tissue microarrays, can be loaded into trays, racks, or carousels. In fact, larger scanners may have a "hotel" that holds many slide trays. There are 


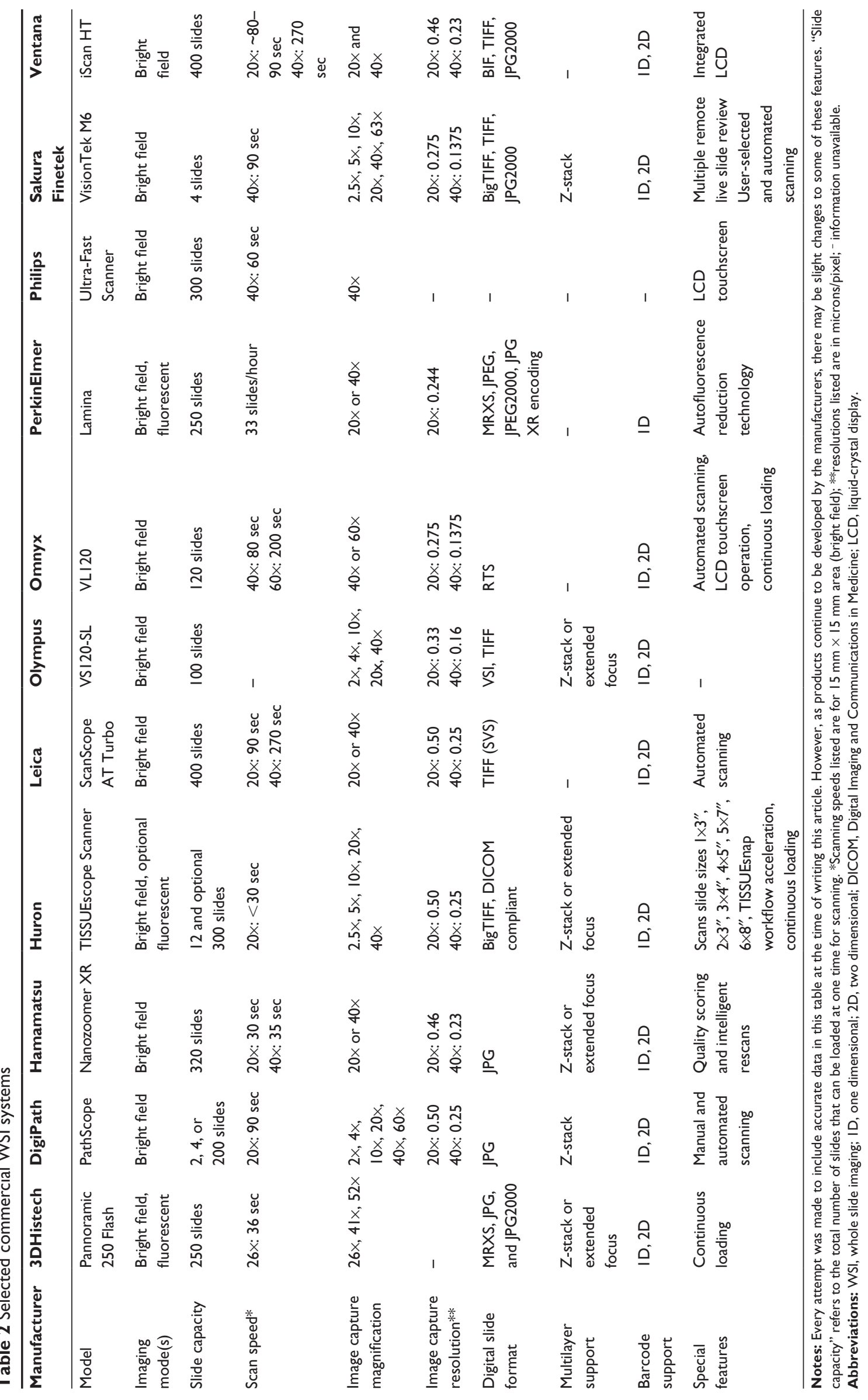


WSI scanners (Figure 2) designed to scan whole mount glass slides (eg, $8^{\prime \prime} \times 6^{\prime \prime}$ slides, such as brain or complete prostate gland sections). It is important to note that some glass slides (eg, very thick or broken slides) may fail automated scanning and may need to be reloaded more than once to allow

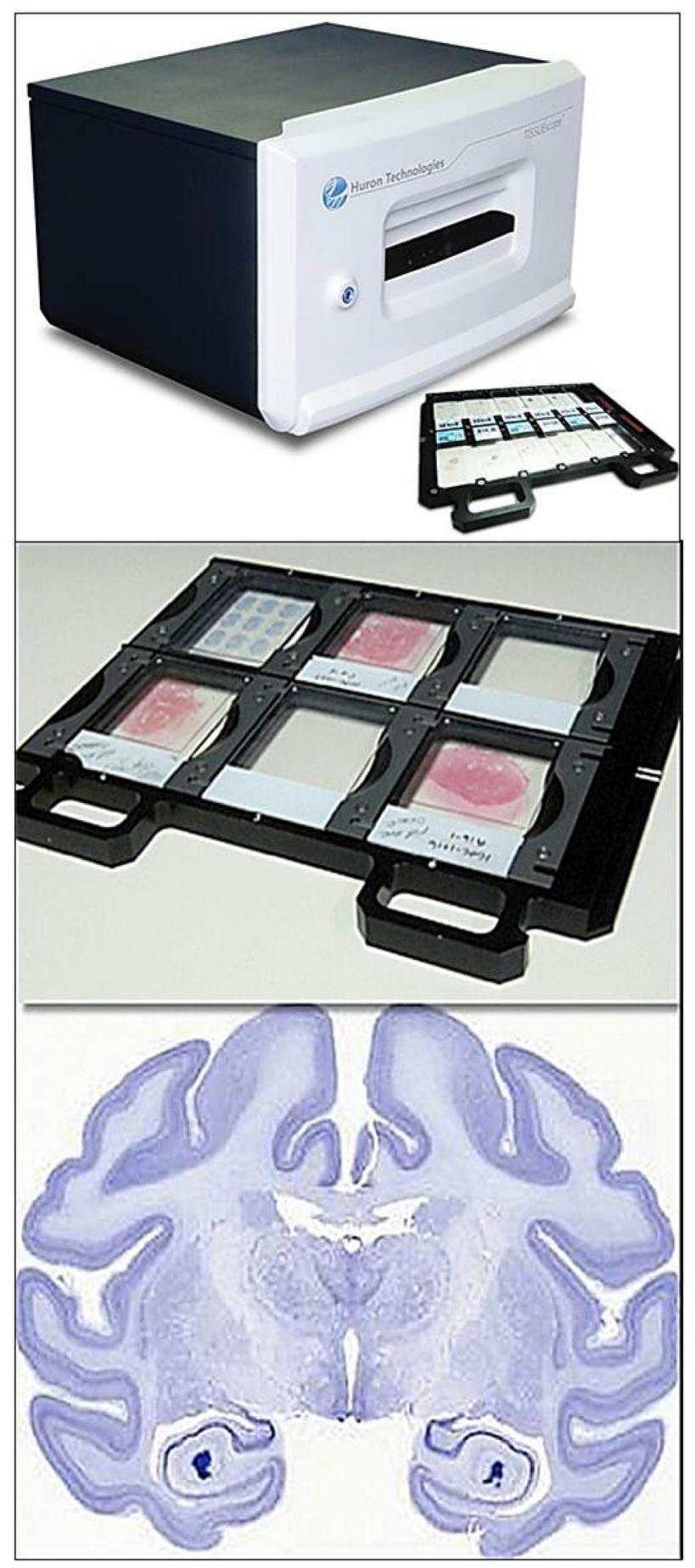

Figure 2 (Top) Huron TISSUEscope scanner that is capable of digitizing a variety of slides ranging from regular $3^{\prime \prime} \times I^{\prime \prime}(75 \mathrm{~mm} \times 25 \mathrm{~mm})$ glass slides to (Middle) glass slides as large as 8" $\times 6$ " $(200 \mathrm{~mm} \times 150 \mathrm{~mm}$ ) (whole slide imaging). (Bottom) Digital whole mount brain image. (Images courtesy of Huron). manual scans. This failure to scan and/or rescan rate is an important factor for customers to consider when purchasing a scanner. Some scanners offer an integrated rescanning feature (eg, NanoZoomer-XR).

A whole slide scanner is, in essence, a microscope under robotic and computer control. This is attached to a highly specialized camera(s) containing advanced optical sensors. The essential components of a WSI scanner, no matter how complex, include the following: 1) a microscope with lens objectives, 2) light source (bright field and/or fluorescent), 3 ) robotics to load and move glass slides around, 4) one or more digital cameras for image capture, 5) a computer, and 6) software to manipulate, manage, and view digital slides. Some devices have dynamic prefocusing functionality, which utilizes one camera to focus and another for scanning. This helps speed up the scanning process. Only a limited number of scanners offer both dry scanning and oil immersion, such as the Aperio CSO scanner designed for hematopathology and microbiology. The robotics in these units are capable of traversing glass slides at speeds upwards of $180 \mathrm{~mm} / \mathrm{s}$. Robotics are key to avoid breaking slides, for stage accuracy, and for dependable objective switching. Not all scanners may be wet slide compatible or are able to scan slides without coverslips. WSI instruments that require glass slides to be loaded in a vertical position (as opposed to lying flat) are less likely to be able to accommodate wet slides with recently applied mounting medium (eg, Leica ScanScope). Certain devices (eg, VisionTek) require the glass slide-coverslip interface for focusing when scanning slides. Modern WSI devices may also incorporate nontraditional optics and illumination techniques (eg, confocal microscopy). ${ }^{1,16,17}$

Slides can be scanned manually or automatically (ie, walk-away functionality). Several WSI instruments do batch scanning (ie, scan one slide at a time) and continuous or random access processing (ie, slides can be uploaded while another is being scanned). Most devices are able to read one-dimensional and two-dimensional barcodes on glass slides. Scanning speeds vary from under 1 minute to around 1-3 minutes per slide, depending on the objective magnification and number of z-stacks (plane of focus) acquired. One can choose to scan an entire glass slide or, if desired, a preselected region of interest on the slide. Certain WSI scanners are able to digitize a glass slide at various z-axes (vertical focal planes), thereby generating a multiplane image that upon viewing simulates the fine focus control of a conventional microscope. Z-stacking (described later) is a desirable feature when viewing cytology slides. 
After digital data are captured via the camera's chargecoupled device (CCD), a computer employs specialized imaging software to generate a virtual slide. Two commonly employed methods for accomplishing this task are tile-based and line-based scanning (Figure 3).

- Tile-based scanning utilizes a robotics-controlled motorized slide stage to obtain large numbers of square image frames, which are assembled together into a mosaic pattern. There is typically $2 \%-5 \%$ overlap in a given tile, thanks to the exceedingly precise saccadic movement of the slide stage. The CCD-captured tiles are then "autocorrelated" with each other to ensure proper alignment, at which point they are "stitched" together in a single, massive seamless image.

- Line-based scanning relies on a servomotor-based slide stage that moves in a jitter-free linear fashion along a single axis of acquisition. After numerous sequential passes at different locations on the slide, a group of images is produced in the form of long, uninterrupted strips or lines. This method of slide acquisition greatly simplifies the image alignment process, since the number of lines/tiles, and degrees of freedom associated with each, that are needed is significantly reduced.

While the computational challenge of tiling systems is much more complex than that of a line-based system, this is less of an issue with current technology, as most WSI scanners today employ computers with superior central processing units, utilize light-emitting diode (LED)-based strobe illumination to eliminate motion blur, incorporate nearly 100 frame-persecond cameras, have improved stage technology (robotics), and use advanced image acquisition algorithms. Some vendors have exploited alternative image acquisition methods such as an array microscope or independent dual sensor scanning (or continuous autofocus), which decouples image acquisition from focusing in order to promote rapid scanning. ${ }^{18}$

The scanner's image resolution (ie, sharpness) is determined by the microscope objective used for scanning (eg, $\times 20, \times 40)$, the numerical aperture of the objective (average numerical aperture used is 0.75 ), and the quality of the camera's photosensors (ie, CCD). Although a digital image can be viewed at any magnification, enlarging an image beyond the true resolution will result in pixilation, causing the image to appear out of focus. Increasing image detail results in increasing file sizes. A typical virtual slide scanned at $\times 40$ may produce a file several gigabytes large. Fortunately, pathology has benefited from Moore's law and its correlates, so while increasingly enormous WSI files are being produced, the storage capacity and speed of computers to handle these files have appropriately increased as well. According to the American Telemedicine Association clinical guidelines for telepathology, it is recommended that adequate storage capacity should be in place if images are to be retained, manipulated, and retrieved. ${ }^{19}$ These guidelines also recommend that laboratories employ a data management system that permits short- and long-term image storage. Redundancy and backups of stored images are also important aspects of any storage solution. Based upon experience from pathology laboratories that have gone fully digital, the amount of storage needed for a fully digital pathology laboratory remains a significant obstacle, despite using lossy compression. Stathonikos et a ${ }^{19}{ }^{19}$ utilized storage tiers to keep their lab's whole slide images, with the top tier serving as a fast disk-based solution and the lower tier a tape archive where all digital slides were copied after a certain period of time. In their laboratory, slides that are scanned initially for diagnostic work get stored in short-term storage, which facilitates fast disk-based solutions for less latency in writing and retrieving. Thereafter, slides needed for second opinions or consultations are entered into longterm storage. This Dutch laboratory has also developed a procedure to purge and retain slides. Digital slides that reside in their short-term storage "expire" after a period of time and are subsequently deleted. Slides that end up in their long-term storage are retained for a longer fixed period of
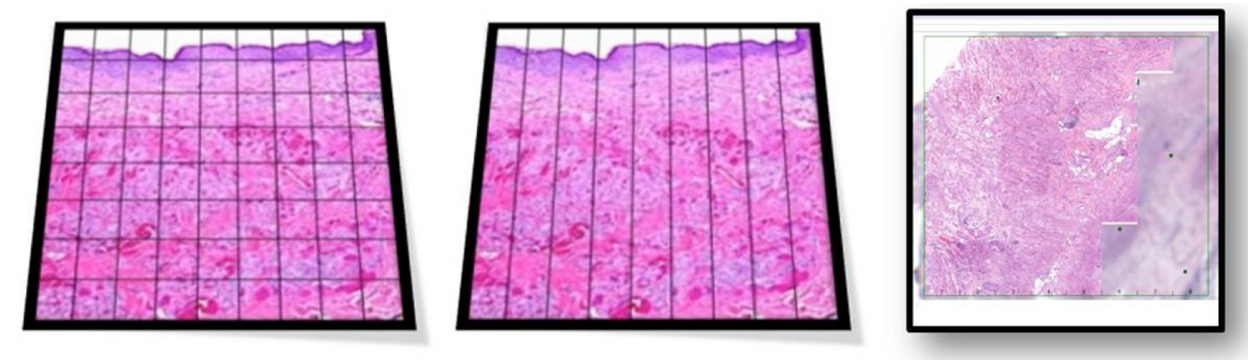

Figure 3 (Left) Tile-based and (Middle) line-based scanning methods. (Right) Line-based scanning of an actual glass slide is shown while in progress. 
time, typically until a diagnostic procedure is concluded. Their educational and research slides are kept indefinitely and moved to permanent storage.

\section{Software}

Software is used for image acquisition (eg, automatic tissue detection, focus calibration, compression) and viewing images and may include applications that facilitate image management, sharing, and image analysis. Some scanners have automated quality scoring of their scanned images. Others offer user interactive software to select the region of a slide to be scanned, adjust white balancing, and perform focusing. After a slide is scanned, file formatting is required to mitigate the massive size of the acquired raw, uncompressed whole slide image file. For example, a typical virtual slide that is 1,600 megapixels would require about 4.6 GB of memory. ${ }^{10}$

The file size can vary greatly among vendors, which will impact storage costs. WSI files are significantly larger than digital image files routinely used by other health care specialties such as radiology. ${ }^{20,21}$ Therefore, compressiondecompression methods, both "lossy" (eg, JPEG2000) and "lossless" (eg, TIFF) types, are employed to archive virtual slides. Typically, these are stored as thousands of image files spanning multiple folders. These files are then constructed into a multilayered "pyramid", enabling optimized realtime viewing across multiple resolutions (ie, $\times 4, \times 20$, etc) instantaneously. Thus, for any given digital magnification, an equal or higher magnification is utilized as the data source. Consequently, decreased computational burden is required to traverse large WSI files. ${ }^{2}$ The "pyramid" encoding model produces a conserved field of view and tile size (or pixel count). The former is favored due to current limitations and monitor display technology, and also since it most accurately replicates the manner of slide viewing that conventional microscopy offers (ie, increasing power/magnification increases the resolution).

Software utilized to navigate digital slides is commonly referred to as an image viewer. It allows users to view and navigate (pan and zoom) virtual slides on a digital screen, reproducing the traditional light microscopy experience in digital format. ${ }^{14}$ WSI viewers enable users to navigate virtual slides at various magnifications. Viewers also offer numerous secondary functions not currently offered by traditional microscopes. These additional functions range from basic offerings like viewing slides at unconventional magnifications (ie, $1 \times$ ) to annotation tools (eg, measurements, overlay text/objects) and more sophisticated features such as image analysis. Some systems also offer teleconferencing tools for simultaneous virtual slide viewing by multiple remote users. Certain image viewers allow simultaneous view of many slides with synchronized navigation. Image analysis software and open source tools (eg, ImageJ) may fail to open very large virtual slides. Fortunately, some investigators have developed cross-platform open source software tools (eg, NDPITools, NDPI-Splitter, Snapshot Creator) to overcome these limitations. ${ }^{22,23}$ NDPITools, for example, allows digital images in NDPI format to be converted to standard TIFF files by creating mosaics (ie, dividing huge images into smaller ones). By splitting large images, such tools facilitate image analysis. The development of other vendor-neutral software is discussed later. Not all viewers are compatible with mobile device use (eg, iPad). Web-based software provided by some vendors supports the deployment of local and cloud-based solutions. At present, not all scanners have software that easily integrates with laboratory information systems.

\section{Clinical applications}

WSI has been used for a wide variety of clinical purposes, including telepathology for primary diagnosis, consultation (second opinions), and remotely interpreting frozen sections. Whole slide images have also been used for remotely viewing immunostains, showcasing pathology slides at tumor boards, saving selected consult slides before they are returned, archiving slides before they get sacrificed to use cellular material for molecular studies, and performing image analysis. Several authors have reported their outcomes using WSI for both primary and secondary (consultation) diagnosis. In general, these publications show good diagnostic concordance between WSI and glass slides. ${ }^{24-31}$ While most of these papers show that diagnoses can indeed be rendered by WSI, an error rate of approximately $1 \%-5 \%$ for WSI does exist. It should be noted, however, that there is also a low baseline discrepancy rate even for glass-to-glass slide review. Discrepancies between WSI and glass slide review have been ascribed mostly to inferior image quality and the user's lack of WSI experience. Moreover, specific microscopic details (eg, mitotic figures, eosinophils) were reported to be difficult to identify. The time required to review digital slides seems to be a recurring theme in many of these papers. Since smaller specimens (eg, biopsies) occupy less area on a slide, they are generally easier to scan. It takes less time to digitize small areas of interest than an entire glass slide. Also, with large specimens, tissue sections on the slide may extend beyond the coverslip, making it hard to focus. However, it is important to be aware that very small pieces of tissue may not always 
be captured with WSI, especially with faint staining. Studies have been published demonstrating that WSI can be effectively used for diagnostic cases where a minimum amount of tissue (eg, needle biopsy) is available. ${ }^{32}$

Since 2006, the University Health Network in Toronto, Canada, implemented WSI telepathology for remote, primary frozen-section diagnoses of small neurosurgical specimens $(<10 \mathrm{~mm})$. In their experience, smaller specimens proved more amenable for the purposes of remote telepathology: $>90 \%$ of specimens were single pieces of tissue that could be entirely embedded, thus simplifying the workflow. By 2011, this institution had logged more than 2,000 cases with a $\leq 5 \%$ deferral rate and $\leq 2 \%$ discrepancy rate when comparing WSI diagnoses with conventional light microscopy diagnoses. ${ }^{4}$ At Kalmar County Hospital in Linköping, Sweden, ergonomic reasons served as the primary catalyst for employing WSI. This program has displayed continued success with large-scale WSI implementation for routine diagnostic work. WSI has been deployed for virtually all histopathology spanning two separate laboratories, which have cumulatively scanned more than half a million slides as of 2013. ${ }^{24}$ In 2010, a medium-sized Dutch laboratory at Atrium Medical Center in Heerlen, the Netherlands, scanned around $20 \%$ of their surgical pathology cases and reported an $82.1 \%$ concordance rate between WSI and glass slide diagnoses. ${ }^{33}$ Many other studies have likewise verified these findings, promoting the use of WSI for diagnostic purposes. Systems capable of digitizing entire peripheral blood smears (eg, CellaVision Automated Digital System ${ }^{\circledR}$ ) have also emerged as a novel mechanism to automatically locate and preclassify digital images of blood cells, as well as facilitate their remote interpretation (ie, telehematology). ${ }^{25}$

WSI is a useful tool for cytopathology. However, it is currently being used more for educational purposes than for routine diagnostic work. ${ }^{34}$ This is because scanning cytology slides is difficult if they contain thick smears or if specimens have three-dimensional (3D) cell groups (eg, Pap tests). This may explain why some WSI-related cytopathology studies have highlighted the underperformance of virtual slides compared with glass slides when images were captured over a narrow focal range..$^{35}$ To overcome this "focusing" challenge, some WSI scanners provide z-axis scanning. Z-stacking involves scanning a glass slide at different focal planes along the vertical z-axis and stacking the images on top of each other to produce a composite multiplane image. ${ }^{36,37}$ Table 2 lists some WSI scanners that offer z-stacking capability. Z-stack scanning, however, takes longer and produces larger digital files. An alternative is to use an extended focusing algorithm.
With this method, multiple smaller scanned images (each at an optimal focal plane) get assembled into a composite image. These are quicker to acquire and of smaller file size than multiplane virtual images. Another challenge in cytology is the need to effectively, easily, and systematically screen an entire slide. Screening depends largely on the viewing software and features that facilitate navigation and annotation and that confirm complete slide coverage. Using a computer mouse for this purpose is tedious. Hence, more studies are needed to evaluate alternative methods of navigating digital slides, such as the use of trackballs, touch pads, gaming station controls, touchscreen monitors, and powerwalls. One study to date has reported success using a prototype ultrahigh-speed WSI viewer based on the Sony PlayStation ${ }^{\circledR} 3$ with wireless controllers. $^{38}$

WSI provides a platform to employ image algorithms that can accurately and reproducibly analyze immunohistochemical studies. Fine et $\mathrm{al}^{39}$ investigated the role of WSI in immunohistochemistry as early as 2006. Others have since demonstrated similarly promising results. Today, WSI platforms have become widely exploited in mammary biomarker analysis (ie, HER2/Neu, estrogen receptor, progesterone receptor, and Ki-67 quantification). ${ }^{40}$ WSI also enables the analysis of immunofluorescent studies. Compared with immunohistochemistry, immunofluorescence offers more reproducible quantification of labeled proteins and multiplexing, both of which facilitate automated analysis. WSI is also amenable to multispectral imaging, which can provide invaluable spectral-spatial information. ${ }^{41}$ Additional WSI-related technologies currently under investigation include automated image analysis tools for computer-assisted diagnosis. ${ }^{41-43}$ Combining WSI with image analysis tools allows users to leverage technology to perform tasks that were previously too cumbersome or even impossible for humans to undertake manually. Examples include:

- high-throughput morphologic analysis of cases to quantitatively and reproducibly measure histologic structures such as tumors; ${ }^{44}$

- automated grading of tumors to reduce variability encountered with manual grading; ${ }^{45}$ and

- automated selection of desired regions of interest, such as hot spots (most active areas in proliferative rate). ${ }^{46}$

\section{Clinical validation}

Validation of WSI is important to evaluate the performance of a WSI system for intended clinical use. For WSI, validation refers to the demonstration of equivalent diagnostic performance between digital slides and glass slides examined 
using a conventional light microscope. A clinical validation study in the laboratory requires documentation of the process involved and the results obtained. The results of a validation study comparing digital slides with glass slides typically measure diagnostic concordance between these two modalities. The study design, technology used, and user training may all influence concordance. A very complicated study that uses low-end computers and pathologists inexperienced at reading digital images may negatively impact concordance. Of note, even glass-to-glass slide comparative studies can yield discrepancies due to observer variability and increasing case difficulty. Several guidelines have been developed that address WSI validation, including the College of American Pathologists (CAP) recommendations for validating WSI for diagnostic purposes. ${ }^{47}$ According to the CAP, validation of the entire WSI system that involves trained pathologists should be performed in a manner that emulates the laboratory's actual clinical environment. The CAP recommends including at least 60 routine cases per application to assess intraobserver diagnostic concordance between digitized and glass slides, viewed at least 2 weeks apart. The cases selected, which may each include more than one slide, should reflect the spectrum and complexity of specimen types and diagnoses likely to be encountered during routine operation.

\section{Nonclinical applications}

WSI is frequently used for numerous educational activities, including multidisciplinary graduate and professional education, virtual tracking/tutoring, performance improvement programs, and medical examinations, and is being incorporated as "illustrations" in journal articles and textbooks. ${ }^{48-55}$ In these settings, virtual slides offer several advantages over conventional glass slides. Digital slides are more interactive, instantaneously available to multiple remote users, can be easily annotated, and promote standardization of training materials (ie, identical slide sets are available for all trainees). For proficiency testing, digital slides are logistically easier to send to multiple sites than glass slides. Glass slides on the other hand require expensive light microscopes, physically degrade over time (eg, loss of staining), and cannot be instantaneously viewed or shared with multiple remote users. Furthermore, with glass slides, certain types of cases are often not incorporated into teaching sets, such as exotic/rare specimens, cytology slides, and small biopsies with scant amounts of material where recuts cannot be obtained, as well as consult slides that must be returned. With WSI platforms, the highestquality slides of the most representative sections can easily be archived for educational purposes in a virtual slide format that will not fade, break, or get lost. However, current limitations of WSI also hold true in the educational setting. Indeed, many users are dissatisfied with current viewing speeds and find viewing software counterintuitive to use. Also, there may be an annoying lag when viewing images, especially when working with massive file sizes over slow networks.

WSI is slowly becoming more relevant for clinical trials. Currently, clinical trials utilize clinicopathological review (aka "central pathology review") to ensure proper classification of cases and lower diagnosis discrepancy rates and to unify target patient populations within the trial inclusion criteria. Many of these trials rely on physically mailing slides to different centers for examination by conventional light microscopy, which takes time and impedes the trial. WSI provides a dramatically streamlined alternative, which substantially reduces the turnaround time required for multiple slide reviews. ${ }^{56}$ With a similar approach, pathology and biomedical communities could reap great benefits by using WSI technology to create enormous image repositories. ${ }^{57,58}$ Large WSI repositories could enable increased peer review of scientific studies and validation of published virtual slide collections and create valuable data for concordance studies and image analysis algorithm development.

\section{Recent advances}

On the clinical front, WSI has instigated several global digital pathology networks, allowing users around the world to connect and leverage the internet and cloud-based solutions. Researchers and WSI vendors are also vigorously working to improve WSI platforms, develop new technology, and create "killer app" functionality. Some recent advances include the use of a high-definition hematoxylin and eosin test for digital pathology, ${ }^{59}$ color calibration slide to promote color standardization in WSI, ${ }^{60}$ and vendor-neutral viewers (eg, OpenSlide). ${ }^{61}$ Tissue folds have a negative impact on the quality of virtual slide images and may interfere with image analysis algorithms. ${ }^{62,63}$ Bautista and Yagi ${ }^{63}$ developed a solution to overcome tissue fold artifacts by way of color enhancement techniques. Researchers have created software (eg, Histostitcher) that generates a pseudo whole mount section by stitching together smaller individual images. ${ }^{64}$ This is especially helpful if one does not have the capability to prepare and/or digitize whole mounts. Software is also available to align whole slide images of serial sections. This allows users to perform virtual double staining and colocalize biomarkers. The introduction of sophisticated software tools that use serial whole slide images to generate and manipulate 3D structures has many applications. With 
3D images, researchers are able to better reconstruct and visualize structures, determine positional relationships between areas of interest and their surrounding structures, as well as obtain new measurements (eg, volumetric data). ${ }^{65}$ Better integration of WSI with clinical systems is needed to improve the widespread adoption of digital pathology. Some laboratories have successfully accomplished such integration. For example, in collaboration with several vendors, the Department of Pathology at the Washington University School of Medicine in the US established a model of "one-stop shopping" by developing an automatic in-house interface between their lab information system and WSI imaging software ${ }^{66}$ Bioinformatics solutions are being created that support the integration of heterogeneous datasets including clinical, next-generation sequencing, and imaging data. ${ }^{67}$ Novel whole slide image acquisition methods using innovative optics and also mobile devices have significant implications for WSI. For example, the Fourier pytchographic microscopy method has been used to rapidly stitch together thousands of low-resolution images to produce a wide-field, high-resolution image. ${ }^{68}$ This technology could drastically reduce the amount of time needed to scan a whole slide by several orders of magnitude.

\section{Conclusion}

WSI technology has matured enormously. Whole slide images have offered the pathology community novel clinical, nonclinical, and research image-related applications. WSI platforms have the potential to improve diagnostic accuracy, increase workflow efficiency, balance workloads, better integrate images with information systems, and financially enhance return on investment. ${ }^{69}$ As more image analysis algorithms and computer-assisted diagnosis tools get developed and validated for clinical use, they will empower pathologists to become more efficient, precise, and reproducible at quantifying prognostic biomarkers like HER2. Standardization is needed for preimaging steps (eg, consistent staining, optimal slide preparation without artifacts), image acquisition (eg, optimal resolution, number of z-stacks, and interval space), postimaging processes (eg, color calibration), and sharing/transmission of digital images (eg, interoperable file formats, vendor agnostic viewers). Albeit slow, efforts with Digital Imaging and Communications in Medicine (DICOM) are underway to help standardize the use of WSI in pathology. ${ }^{70}$ However, the adoption of WSI by pathologists worldwide has been slow for several reasons, including limiting technology, image quality, shortcomings to scan all materials (eg, cytology, microbiology), the cost of these systems and digital slide storage, their inability to handle high-throughput routine work, regulatory barriers in certain countries, user-unfriendly ergonomics, and pathologists' reluctance to use WSI. ${ }^{71}$ Nonetheless, we believe that all of these challenges are solvable and anticipate that WSI will continue to transform the practice of pathology and hopefully catalyze further novel applications that will benefit our field and the patients we serve.

\section{Acknowledgments}

We would like to thank Jon Duboy and Andrew Lesniak from UPMC for their technical advice. We also thank those vendors that provided us with images and information about their WSI scanners.

\section{Disclosure}

Dr Parwani is an advisor to Omnyx on their pathologist team. Dr Parwani has received research support from Omnyx. The other authors report no conflicts of interest in this work.

\section{References}

1. Parwani AV, Feldman M, Balis U, et al. Telepathology. In: Pantanowitz L, Tuthill MJ, Balis U, editors. Pathology Informatics: Theory and Practice. Canada: ASCP; 2012:261-272.

2. Parwani AV, Feldman M, Balis U, et al. Digital imaging. In: Pantanowitz L, Tuthill MJ, Balis U, editors. Pathology Informatics: Theory and Practice. Canada: ASCP; 2012:231-260.

3. Wiley CA, Murdoch G, Parwani A, et al. Interinstitutional and interstate teleneuropathology. J Pathol Inform. 2011;2:21.

4. Evans AJ, Chetty R, Clarke BA, et al. Primary frozen section diagnosis by robotic microscopy and virtual slide telepathology: the University Health Network experience. Hum Pathol. 2009;40(8):1070-1081.

5. Gifford AJ, Colebatch AJ, Litkouhi S, et al. Remote frozen section examination of breast sentinel lymph nodes by telepathology. ANZ J Surg. 2012;82:803-808.

6. Leong FJ, Leong AS. Digital photography in anatomical pathology. J Postgrad Med. 2004;50:62-69.

7. Pantanowitz L, Valenstein PN, Evans AJ, et al. Review of the current state of whole slide imaging in pathology. J Pathol Inform. 2011;2:36

8. Yagi Y, Gilbertson JR. Digital imaging in pathology: the case for standardization. J Telemed Telecare. 2005;11(3):109-116.

9. Wilbur DC. Digital cytology: current state of the art and prospects for the future. Acta Cytol. 2011;55(3):227-238.

10. Park S, Pantanowitz L, Parwani AV. Digital imaging in pathology. Clin Lab Med. 2012;32(4):557-584.

11. Dunn BE, Almagro UA, Choi H, et al. Dynamic-robotic telepathology: Department of Veterans Affairs feasibility study. Hum Pathol. 1997;28: 8-12.

12. Ferreira R, Moon B, Humphries, Sussman A, Saltz J, Miller R, Demarzo A. The virtual microscope. AMIA Ann Fall Symp. 1996: 449-453.

13. Gilbertson JR, Patel AA, Yagi Y. Clinical slide digitization - whole slide imaging in clinical practice. In: Gu J, Olgilive RW, et al, editors. Virtual Microscopy and Virtual Slide in Teaching, Diagnosis, and Research. Boca Rotan, FL: Taylor and Francis; 2005.

14. Rojo MG, Garcia GB, Mateos CP, et al. Critical comparison of 31 commercially available digital slide systems in pathology. Int J Surg Pathol. 2006;14(4):285-305. 
15. Soenksen DG. A fully integrated virtual microscopy system for ultrafast slide scanning, storage, viewing, annotation, and processing. Arch Pathol Lab Med. 2003;127:805.

16. Amin W, Srintrapun SJ, Parwani AV. Automated whole slide imaging. Expert Opin Med Diagn. 2008;2(10):1173-1181.

17. Pantanowitz L, Dickinson K, Evans AJ, et al. American Telemedicine Association clinical guidelines for telepathology. J Pathol Inform. 2014;5:39.

18. Weinstein RS, Descour MR, Liang C, et al. An array microscope for ultrarapid virtual slide processing and telepathology. Design, fabrication, and validation study. Hum Pathol. 2004;35(11):1303-1314.

19. Stathonikos N, Veta M, Huisman A, van Diest PJ. Going fully digital: perspective of a Dutch academic pathology lab. J Pathol Inform. $2013 ; 4: 15$

20. Hipp JD, Fernandez A, Compton CC, et al. Why a pathology image should not be considered as a radiology image. J Pathol Inform. 2011;2:26

21. Montalto MC. Pathology re-imagined: The history of digital radiology and the future of anatomic pathology. Arch Pathol Lab Med. 2008;123(5):764-765

22. Deroulers C, Ameisen D, Badoual M, Gerin C, Granier A, Lartaud M. Analyzing huge pathology images with open source software. Diagn Pathol. 2013;8:92.

23. Khushi M, Edwards G, de Marcos DA, Carpenter JE, Graham JD, Clarke CL. Open source tools for management and archiving of digital microscopy data to allow integration with patient pathology and treatment information. Diagn Pathol. 2013;8:22.

24. Thorstenson S, Molin J, Lundström C. Implementation of large-scale routine diagnostics using whole slide imaging in Sweden: digital pathology experiences 2006-2013. J Pathol Inform. 2014;5:14.

25. Pantanowitz L, Wiley CA, Demetris A, et al. Experience with multimodality telepathology at the University of Pittsburgh Medical Center. J Pathol Inform. 2012;3:45.

26. Al Habeeb A, Evans A, Ghazarian D. Virtual microscopy using whole-slide imaging as an enabler for teledermatopathology: a paired consultant validation study. J Pathol Inform. 2012;3:2.

27. Al-Janabi S, Huisman A, Vink A, et al. Whole slide images for primary diagnostics in dermatopathology: a feasibility study. J Clin Pathol. 2012;65:152-158.

28. Nielsen PS, Lindebjerg J, Rasmussen J, et al. Virtual microscopy: an evaluation of its validity and diagnostic performance in routine histologic diagnosis of skin tumors. Hum Pathol. 2010;41(12):1770-1776.

29. Leinweber B, Massone C, Kodama K, et al. Teledermatopathology: a controlled study about diagnostic validity and technical requirements for digital transmission. Am J Dermatopathol. 2006;28(5):413-416.

30. Massone C, Soyer HP, Lozzi GP, et al. Feasibility and diagnostic agreement in teledermatopathology using a virtual slide system. Hum Pathol. 2007;38(4):546-554.

31. Koch LH, Lampros JN, Delong LK, Chen SC, Woosley JT, Hood AF. Randomized comparison of virtual microscopy and traditional glass microscopy in diagnostic accuracy among dermatology and pathology residents. Hum Pathol. 2009;40(5):662-667.

32. Campbell WS, Hinrichs SH, Lele SM, et al. Whole slide imaging diagnostic concordance with light microscopy for breast needle biopsies. Hum Pathol. 2014;45:1713-1721.

33. Al-Janabi S, Huisman A, Nap M, Clarijs R, van Diest PJ. Whole slide images as a platform for initial diagnostics in histopathology in a medium-sized routine laboratory. J Clin Pathol. 2012;65(12): 1107-1111.

34. House JC, Henderson-Jackson EB, Johnson JO, et al. Diagnostic digital cytopathology: are we ready yet? J Pathol Inform. 2013;4:28.

35. Evered A, Dudding N. Accuracy and perceptions of virtual microscopy compared with glass slide microscopy in cervical cytology. Cytopathology. 2011;22(2):82-87.

36. Alsharif M, Carlo-Demovich J, Massey C, et al. Telecytopathology for immediate evaluation of fine-needle aspiration specimens. Cancer Cytopathol. 2010;118(3):119-126.
37. Kaplan KJ. Telecytopathology for immediate evaluation of fine-needle aspiration specimens. Cancer Cytopathol. 2010;118:115-118.

38. Yagi Y, Yoshioka S, Kyusojin H, et al. An ultra-high speed whole slide image viewing system. Anal Cell Pathol (Amst). 2012;35:65-73.

39. Fine JL, Grzybicki DM, Silowash R, et al. Evaluation of whole slide image immunohistochemistry interpretation in challenging prostate needle biopsies. Hum Pathol. 2008;39(4):564-572.

40. Lloyd MC, Allam-Nandyala P, Purohit CN, et al. Using image analysis as a tool for assessment of prognostic and predictive biomarkers for breast cancer: how reliable is it? J Pathol Inform. 2010;1:29.

41. DiFranco MD, O'Hurley G, Kay EW, Watson RW, Cunningham P. Ensemble based system for whole-slide prostate cancer probability mapping using color texture features. Comput Med Imaging Graph. 2011;35:629-645.

42. Samsi S, Krishnamurthy AK, Gurcan MN. An efficient computational framework for the analysis of whole slide images: application to follicular lymphoma immunohistochemistry. J Comput Sci. 2012;3: 269-279.

43. Sertel O, Kong J, Shimada H, Catalyurek UV, Saltz JH, Gurcan MN. Computer-aided prognosis of neuroblastoma on whole-slide images: classification of stromal development. Pattern Recognit. 2009;42: 1093-1103

44. Kong J, Cooper LA, Wang F, Gao J, et al. Machine-based morphologic analysis of glioblastoma using whole-slide pathology images uncovers clinically relevant molecular correlates. PLoS One. 2013;8:e81049.

45. Yeh FC, Parwani AV, Pantanowitz L, Ho C. Automated grading of renal cell carcinoma using whole slide imaging. J Pathol Inform. 2014;5:23.

46. Lu H, Papathomas TG, van Zessen D, et al. Automated Selection of Hotspots (ASH): enhanced automated segmentation and adaptive step finding for Ki67 hotspot detection in adrenal cortical cancer. Diagn Pathol. 2014;9:216.

47. Pantanowitz L, Sinard JH, Henricks WH, et al. Validating whole slide imaging for diagnostic purposes in pathology: guideline from the College of American Pathologists Pathology and Laboratory Quality Center. Arch Pathol Lab Med. 2013;137(12):1710-1722.

48. Blake CA, Lavoie HA, Millette CF. Teaching medical histology at the University of South Carolina School of Medicine: transition to virtual slides and virtual microscopes. Anat Rec B New Anat. 2003;275(1): 196-206.

49. Foster K. Medical education in the digital age: digital whole slide imaging as an e-learning tool. J Pathol Inform. 2010;1:14.

50. Dee FR, Meyerholz DK. Teaching medical pathology in the twenty-first century: virtual microscopy applications. J Vet Med Educ. 2007;34: 431-436.

51. Treanor D, Lim CH, Magee D, Bulpitt A, Quirke P. Tracking with virtual slides: a tool to study diagnostic error in histopathology. Histopathology. 2009;55:37-45.

52. Mello-Thoms C, Pantanowitz L, Parwani A, Ho J, Sharma G, Crowley R. Analysis of digital slide exploration characteristics of expert pathologists. J Pathol Inform. 2010;1:22.

53. Sharma G, Kelly SM, Wiehagen LT, Palekar A, Pantanowitz L, Parwani AV. Implementation of whole slide imaging for multi-site review of performance improvement program (PIP) slides. Arch Path Lab Med. 2011;135:1227-1228.

54. Marchevsky AM, Khurana R, Thomas P, Scharre K, Farias P, Bose S. The use of virtual microscopy for proficiency testing in gynecologic cytopathology: a feasibility study using ScanScope. Arch Pathol Lab Med. 2006;130:349-355

55. Pantanowitz L, Szymas J, Yagi Y, Wilbur D. Whole slide imaging for educational purposes. J Pathol Inform. 2012;3:46.

56. Pawel M, Parwani AV, Piotr K. Central pathology review for phase III clinical trials: the enabling effect of virtual microscopy. Arch Pathol Lab Med. 2013;137:492-495.

57. Hipp JD, Lucas DR, Emmert-Buck MR, Compton CC, Balis UJ. Digital slide repositories for publications: lessons learned from the microarray community. Am J Surg Pathol. 2011;35:783-786. 
58. Hipp JD, Sica J, McKenna B, et al. The need for the pathology community to sponsor a whole slide imaging repository with technical guidance from the pathology informatics community. J Pathol Inform. 2011;2:31

59. Martina JD, Simmons C, Jukic DM. High-definition hematoxylin and eosin staining in a transition to digital pathology. J Pathol Inform. 2011;2:45.

60. Bautista PA, Hashimoto N, Yagi Y. Color standardization in whole slide imaging using a color calibration slide. J Pathol Inform. 2014;5:4.

61. Goode A, Gilbert B, Harkes J, Jukic D, Satyanarayanan M. OpenSlide: a vendor-neutral software foundation for digital pathology. J Pathol Inform. 2013;4:27.

62. Yagi Y, Gilbertson J. A relationship between slide quality and image quality in whole slide imaging. Diagn Pathol. 2008;3:S12.

63. Bautista PA, Yagi Y. Improving the visualization and detection of tissue folds in whole slide images through color enhancement. J Pathol Inform. 2010;1:25.

64. Toth RJ, Shih N, Tomaszewski JE, Feldman MD, et al. Histostitcher ${ }^{\mathrm{TM}}$ : an informatics software platform for reconstructing whole-moun prostate histology using the extensible imaging platform framework. J Pathol Inform. 2014;5:8.
65. Roberts N, Magee D, Song Y, et al. Toward routine use of 3D histopathology as a research tool. Am J Pathol. 2012;180:1835-1842.

66. Isaacs M, Lennerz JK, Yates S, Clermont W, Rossi J, Pfeifer JD. Implementation of whole slide imaging in surgical pathology: a value added approach. J Pathol Inform. 2011;2:39.

67. Dander A, Baldauf M, Sperk M, Pabinger S, Hiltpolt B, Trajanoski Z Personalized Oncology Suite: integrating next-generation sequencing data and whole-slide bioimages. BMC Bioinformatics. 2014;15:306.

68. Zheng G, Horstmeyer R, Yang C. Wide-field, high-resolution Fourier ptychographic microscopy. Nat Photonics. 2013;7(9):739-745.

69. Ho J, Ahlers SM, Stratman C, et al. Can digital pathology result in cost savings? A financial projection for digital pathology implementation at a large integrated health care organization. J Pathol Inform. 2014;5:33

70. Singh R, Chubb L, Pantanowitz L, et al. Standardization in digital pathology: supplement 145 of the DICOM standards. J Pathol Inform. 2011;2:23

71. Parwani AV, Hassell L, Glassy E, et al. Regulatory barriers surrounding the use of whole slide imaging in the United States of America. J Pathol Inform. 2014;5:38.
Pathology and Laboratory Medicine International

\section{Publish your work in this journal}

Pathology and Laboratory Medicine International is a peer-reviewed, open access journal focusing on innovative basic research and translational research related to pathology or human disease. The journal includes original research, updates, case reports, reviews and commentaries on current controversies. The Academic Sponsor

\section{Dovepress}

of this journal is the Chinese American Pathology Association (CAPA). The manuscript management system is completely online and includes a very quick and fair peer-review system. Visit http://www.dovepress.com/testimonials.php to read real quotes from published authors.

Submit your manuscript here: http://www.dovepress.com/pathology-and-laboratory-medicine-international-journal 